\title{
Energetic electrons along the high-latitude magnetopause
}

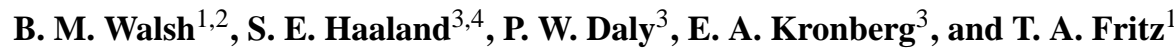 \\ ${ }^{1}$ Center for Space Physics, Boston University, Boston, MA, USA \\ ${ }^{2}$ NASA, Goddard Space Flight Center, Greenbelt, MD, USA \\ ${ }^{3}$ Max Planck Institute for Solar System Research, Lindau, Germany \\ ${ }^{4}$ Department of Physics and Technology, University of Bergen, Bergen, Norway
}

Correspondence to: B. M. Walsh (brian.m.walsh-1@ nasa.gov)

Received: 23 January 2012 - Revised: 18 April 2012 - Accepted: 6 June 2012 - Published: 28 June 2012

\begin{abstract}
A case study is presented to determine the source of the energetic electron layer frequently observed along the high-latitude magnetopause. Measurements by the Cluster spacecraft show bursts of field-aligned electrons occurring during time periods with high potential for dayside reconnection. These properties are compared with the expected signatures from several sources including escape from the exterior cusp, acceleration in a reconnection region, and release from the dayside trapping region through reconnection. The observed properties are most consistent with the electrons being released from the magnetosphere due to reconnection. In this model the electrons would flow along the newly reconnected IMF draped along the magnetopause and propagate along the high-latitude magnetopause. These observations demonstrate an active source for populating the energetic particle layer frequently observed along and just outside the high-latitude magnetopause.
\end{abstract}

Keywords. Magnetospheric physics (Magnetopause, cusp, and boundary layers; Magnetosheath) - Space plasma physics (Charged particle motion and acceleration)

\section{Introduction}

A layer of superthermal ions and electrons has been consistently observed along and just outside the magnetopause. The electron layer has been measured along the equatorial magnetopause on the dayside, the flanks (Mitchell et al., 1987), and extending as far as $60 R_{\mathrm{E}}$ downtail (Meng and Anderson, 1970). Additional observations have detected this electron layer to be a common occurrence at high latitudes as well (Domingo et al., 1977; Zong et al., 2005). At high latitudes poleward of the cusp, Domingo et al. (1977) observed energetic electrons during more than $90 \%$ of the passes of a two year study with the HEOS-2 spacecraft. Further downtail Baker and Stone (1978) found energetic $(E>200 \mathrm{keV})$ electrons present during $97 \%$ of the boundary passes by the IMP-8 spacecraft. Additional properties of the electron population at high latitudes include a positive correlation with geomagnetic indices (Meng and Anderson, 1975; Baker and Stone, 1977), a peak in intensity near the cusp (Formisano and Domingo, 1979), and a continual tailward flow (Baker and Stone, 1978).

From the properties of the particles, it is clear that a significant portion, if not all of this superthermal population, originates from within the magnetosphere. A detailed look at the plasma composition reveals significant amounts of $\mathrm{He}^{+}$(Sonnerup et al., 1981) and $\mathrm{O}^{+}$(Peterson et al., 1982; Sarafopoulos et al., 1999). These ion species originate from within the magnetosphere, so their presence is evidence of magnetospheric particles contributing to this layer. The energy spectrum of the particles along the magnetopause is also consistent with a magnetospheric source (Williams et al., 1979). Lastly, energy dispersion signatures of energetic ions and electrons near the magnetopause in the magnetosheath are consistent with these particles originating in the low latitude boundary layer (Sarafopoulos et al., 2000).

Since the majority of energetic particles along the magnetopause originated within the magnetosphere, understanding the properties of the layer can give insight into the transport mechanisms moving particles through the boundary. At lower latitudes several mechanisms have shown to be active. Reconnection can open previously closed magnetospheric field lines to allow trapped particles to travel out into the magnetosheath (Daly et al., 1984; Fuselier et al., 1995); gradient and curvature drifts can allow particles to drift out of 
the magnetosphere (Roederer, 1967; Sibeck and McEntire, 1988; Shprits et al., 2006); and particle diffusion due to interactions with wave activity can allow particles to travel through the magnetopause (Treumann et al., 1991).

At higher latitudes, poleward of the cusp, there is no significant trapped energetic particle population inside the magnetopause. The magnetospheric lobe region poleward of the cusp is generally cool with low particle fluxes. This limits the mechanisms that could be creating the energetic particle layer. Since there is no source population just inside the magnetopause, particles could not drift out through the boundary here or be released into the boundary layer at high latitudes by local magnetic reconnection. Three potential sources for the energetic electron layer remain however: (1) escape from the exterior cusp; (2) release from the dayside trapping region through reconnection; and (3) electron heating within a reconnection site. Each source is tested in the current study to determine which is most consistent with observations.

The exterior cusp has been shown to be capable of heating electrons to several hundred $\mathrm{keV}$ under certain conditions (Walsh et al., 2010; Nykyri et al., 2012). This energetic electron population is typically peaked at a pitch angle of $90^{\circ}$ and trapped within a magnetic cavity in the exterior cusp. As the magnetic topology of the cusp shifts or the magnetic field strength in the magnetosheath decreases, the magnetic trap could break, releasing this energetic population, potentially flowing out onto the magnetopause as predicted by Formisano and Domingo (1979) and Fritz et al. (2000).

The second source is the dayside trapping region equatorward of the cusp. Dayside reconnection can open magnetospheric field lines and allow particles to flow out from the trapping region. These particles can travel along the newly reconnected IMF draped along the magnetopause and create this energetic particle layer.

The last source once again relies on reconnection but is the heating of electrons up to hundreds of $\mathrm{keV}$ through the reconnection process (Øieroset et al., 2002). These particles would once again flow out along the draped newly reconnected IMF and create a layer of energetic electrons.

\section{Instrumentation}

Observations used in this study were made as part of the Cluster Guest Investigator Programme and were coordinated by the Cluster team. The measurements took advantage of unique orbits and utilized special instrument modes.

The four Cluster spacecraft were launched into an elliptical polar orbit in 2000 with an orbital period of $57 \mathrm{~h}$. As the mission progressed, apogee dropped into the Southern Hemisphere, allowing measurements to be made of the highlatitude magnetopause, poleward of the cusp. Each spacecraft was launched with identical instrument suits.

The low energy electrons from $22 \mathrm{eV}-26.4 \mathrm{keV}$ were measured with PEACE (Johnstone et al., 1997). Energetic elec- trons (41-400 keV) and ions ( $28 \mathrm{keV}-4 \mathrm{MeV})$ were measured with IES and IIMS respectively on the RAPID instrument (Wilken et al., 1997, 2001). The RAPID detector was operating in Nominal Mode 3 during these observations. This allowed for sampling of 9 polar and 16 azimuthal angular bins at spin resolution for each of the 8 electron energy bins. This allowed for full pitch angle distributions to be made for each electron energy channel from $22 \mathrm{eV}-400 \mathrm{keV}$. Measurements with $4 \mathrm{~s}$ spin-resolution were used for both the PEACE and RAPID instruments. Lastly, measurements of the magnetic field with 5 vectors per second were made with FGM (Balogh et al., 1997, 2001).

Measurements from the GEOTAIL Magnetic Field experiment (MGF) (Kokubun et al., 1994) are used to compare with the Cluster observations. The magnetic field measurements are at $3 \mathrm{~s}$ resolution.

\section{Observations}

\subsection{C2 measurements}

On 3 April 2011 all four Cluster spacecraft passed through the high-latitude magnetopause. The measurements were poleward of the cusp and on the dawn side of noon in magnetic local time. Observations of the electron population and magnetic field measured by $\mathrm{C} 2$ are shown in Fig. 1. The boundary layer is identified through a change in the magnetic field vector given in Fig. 1d and e. While inside the boundary layer, the magnetic field is more variable and decreased slightly in magnitude from when the spacecraft is in the adjacent magnetosheath or magnetospheric regions. Inside the magnetosphere (near 02:05 UT), the magnetic field vector points in the negative $B_{\mathrm{x}}, B_{\mathrm{y}}$, and $B_{\mathrm{z}}$ directions as is what one would expect in the lobe on the dawnside in the Southern Hemisphere.

During the boundary crossing, several bursts of enhanced superthermal electron flux were measured. Each burst lasted for between 30 and $120 \mathrm{~s}$. Two of these bursts are outlined in Fig. 1. Burst 1 is focused on in Fig. 1f, g, and h. This enhancement occurs from 01:07:40 to 01:08:15 UT while the spacecraft is in the magnetosheath just outside the boundary layer. The energetic electron population was streaming along the magnetic field line with a peak in pitch angle near $40^{\circ}$. The pitch angle distribution of 41-52 keV electrons during an electron burst is shown in Fig. $1 \mathrm{~g}$ and $\mathrm{h}$. The magnetic field components during this time period indicate these particles are streaming tailward, consistent with previous observations further downtail along the magnetopause (Baker and Stone, 1978). Energetic ions enhancements were also observed during this period and are shown in Fig. 1a.

Burst 2 occurs several minutes later just after the spacecraft enters the boundary layer from 01:21:00-01:23:30 UT. The enhancement shows lower fluxes than the first, but similar properties. The second burst is focused on in Fig. 1i, j, and 


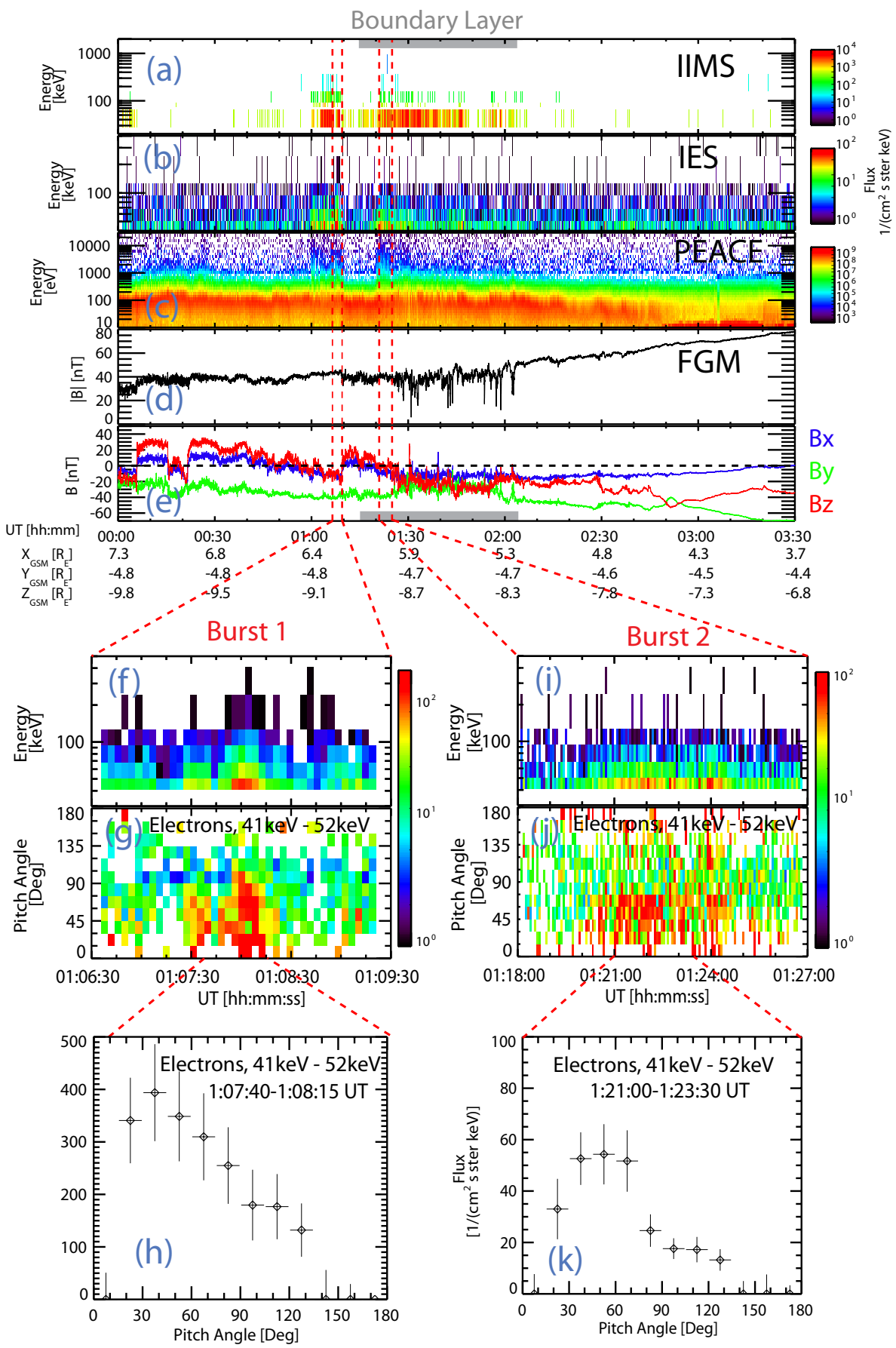

Fig. 1. Electron and magnetic field measurements from $C 2$ during a high-latitude magnetopause crossing are shown. (a) is energetic omnidirectional ions from IIMS on RAPID; (b) is energetic omnidirectional electrons from IES on RAPID; (c) is electron flux from PEACE; and the bottom two panels (d) and (e) are the magnetic field measurements in GSM from FGM. The vertical red bars outline two time periods when bursts of energetic electrons were observed. These two bursts are then zoomed in on in the bottom six plots. Burst 1 is focused on in plots (f), (g), and (h). Panel (f) is omnidirectional energetic electron flux, while (g) and (h) are cuts of the pitch angle distribution for 41-52 keV electrons. Panels (i), (j), and (k) follow the same format as (f), (g), and (h) but for burst 2.

k. Once again, the majority of the energetic electrons demonstrate a field-aligned population traveling tailward with a peak pitch angle near $55^{\circ}$. A few electrons are also observed in a narrow pitch angle range flowing antiparallel to the magnetic field line with pitch angles very close to $180^{\circ}$. The presence of these particles may be due to mirroring from a 

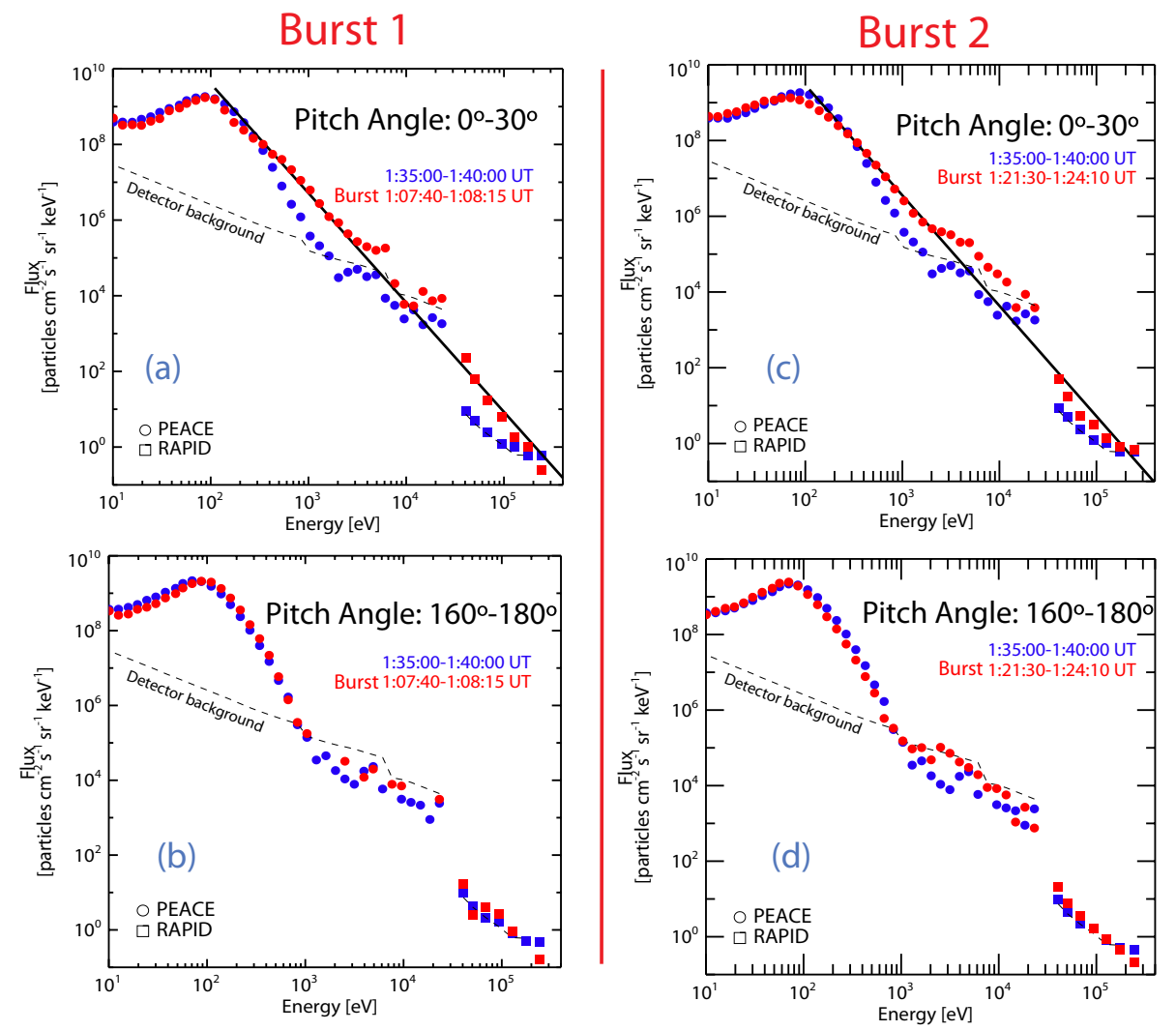

Fig. 2. Electron distributions measured by PEACE and RAPID on $C 2$ are shown for the two bursts and at two pitch angle bins. Each panel compares flux measured during an electron burst (red traces) with a time period when the spacecraft is within the magnetopause boundary layer but did not observe an electron enhancement (blue traces). Measurements by PEACE are shown with circles and those by RAPID are squares. On the left side, panels (a) and (b) are burst 1 while on the right, (c) and (d) are burst 2. Dashed black lines are the background levels for each detector. A power law tail fit to the burst time periods in (a) and (b) is drawn in black. Since the other distributions have many measurements below the background of the detector, a power law is not fit to them.

magnetic maximum on the open field line or some type of scattering effect.

During both bursts the particle distribution indicates an additional electron population being present. Figure 2 shows the particle distribution during the two electron bursts measured by $\mathrm{C} 2$ split into pitch angle bins. Each panel compares a time period during an electron burst (red points) with a time period when the spacecraft was in the boundary layer without an electron burst (blue points). Figure $2 \mathrm{a}$ and $\mathrm{b}$ are from burst 1, while Fig. $2 \mathrm{c}$ and $\mathrm{d}$ are from burst 2 as outlined in Fig. 1. Each plot shows an energy distribution peak near $\sim 100 \mathrm{eV}$, consistent with the presence of electrons from the shocked solar wind in the boundary layer. In the top two panels (field-aligned), the burst time periods show an increase for particles with energy above $\sim 300 \mathrm{eV}$ over the spectra in the non-burst time period. In the bottom two panels, the spectra during both time periods are similar. This means there is little to no enhancement of particle flowing antiparallel to the magnetic field line during the bursts, but an additional population flowing parallel to the magnetic field.
During the burst time period (red symbols), the energetic part of the distribution demonstrates a power law. Given the power law form, Flux $\sim E^{-\gamma}$, the coefficients for burst 1 and burst 2 were $\gamma=2.89$ and $\gamma=2.92$, respectively. These were found through fitting the measurements above the background detector (dashed black lines in Fig. 2). Monitoring energetic electrons $(E>200 \mathrm{keV})$ along the magnetopause further downtail $\left(-10 R_{\mathrm{E}}<X_{\mathrm{SM}}<-40 R_{\mathrm{E}}\right.$ ), Baker and Stone (1978) found a similar power law tail with $\gamma$ ranging from 2 to 4 with a standard value of 3 . This is consistent with the observations presented here and made just poleward of the cusp.

Since Cluster was in the magnetosheath before crossing the magnetopause, the FGM provides measurements of the draped IMF as can be seen in Fig. 1e. The solar wind $B_{\mathrm{Z}}$ component measured by Cluster was primarily northward for $40 \mathrm{~min}$ before the spacecraft reached the boundary layer. Near the boundary layer, the IMF $B_{\mathrm{Z}}$ component turned southward before turning northward again. Throughout the pass there was a strong negative IMF $B_{\mathrm{y}}$ component. 


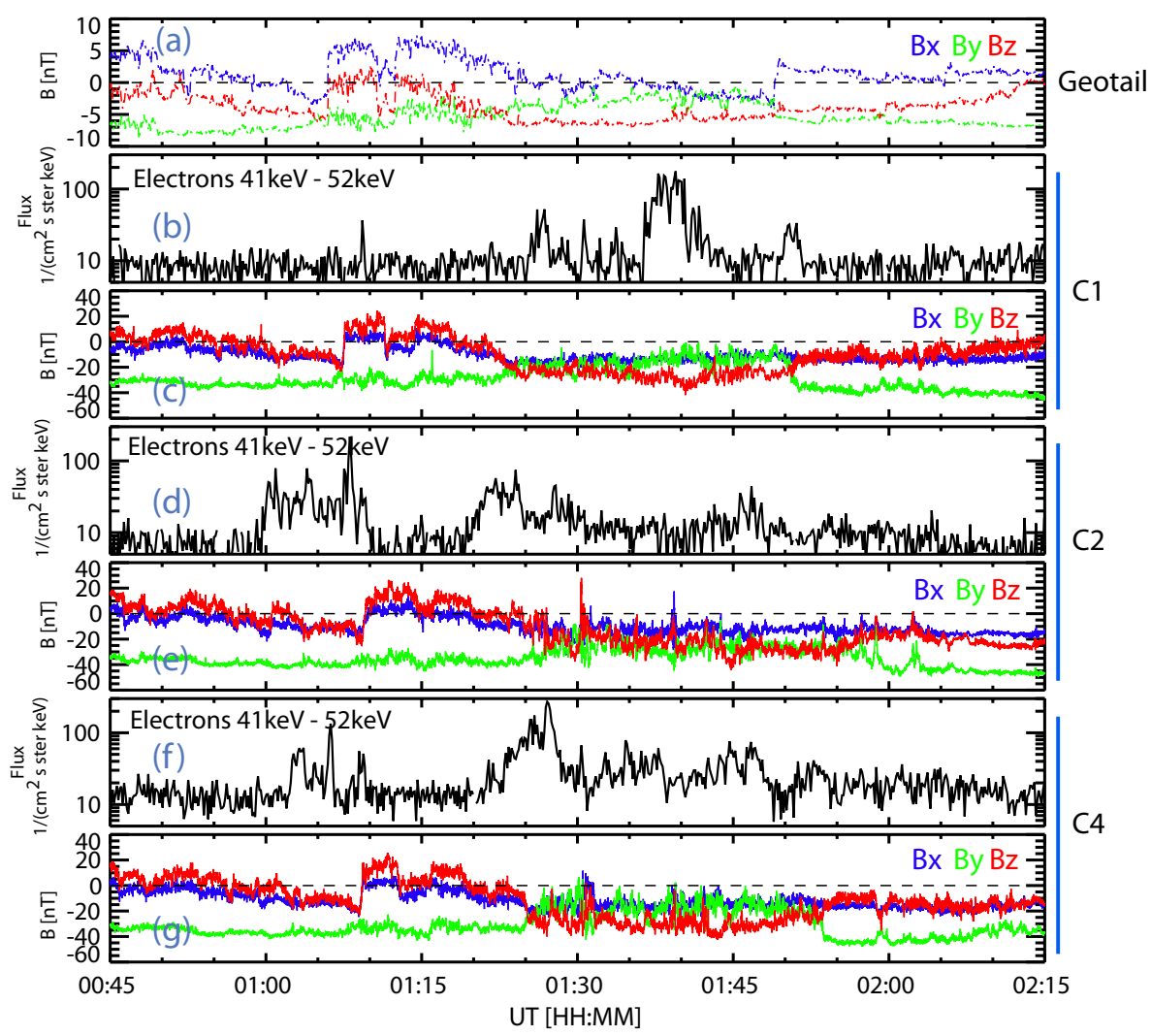

Fig. 3. Cluster and GEOTAIL measurements of the magnetic field components and energetic electrons. GEOTAIL is positioned at $(X, Y, Z)=(8.85,-24.6,-14.2)$ in GSM coordinates. Its measurements are in panel (a). Panels (b), (d), and (f) are omnidirectional electrons measured by RAPID for C1, C2, and C4, respectively. Panels (c), (e), and (g) are magnetic field measured by FGM on C1, C2, and C4, respectively.

\subsection{Multipoint measurements}

The four Cluster spacecraft provide the opportunity to probe possible spatial or temporal features in the electron enhancements. If the spatially separated spacecraft observe an enhancement at the same time, it can be determined that the enhancements are temporal. If multiple spacecraft see a similar electron structure successively as each passes through the boundary, it can be determined that the enhancements are spatial or that there is a layer of energetic electrons present for at least the time periods between the spacecraft passings. To test this possible temporal/spatial nature, energetic electron fluxes from $\mathrm{C} 1, \mathrm{C} 2$, and $\mathrm{C} 4$ are analysed and shown in Fig. 3. The magnetic field measurements from each spacecraft are also presented. The background in the 41$52 \mathrm{keV}$ electron channel from $\mathrm{C} 3$ is too high to be used in this study, so $\mathrm{C} 3$ has been left out. The background is near 60 particles $\mathrm{cm}^{-2} \mathrm{~s}^{-1} \mathrm{ster}^{-1} \mathrm{keV}^{-1}$, which is similar to the level of the enhancements measured by the other spacecraft for this boundary crossing.

To provide more information about the timing of the boundary crossings, the local magnetic field measured by Cluster is compared with the GEOTAIL spacecraft, which was at $(X, Y, Z)=(8.85,-24.6,-14.2) R_{\mathrm{E}}$ in GSM coordinates. Figure 3 compares the magnetic field measurements by Cluster and GEOTAIL as well as electron flux measurements. The magnetic field profiles are similar when both GEOTAIL and Cluster are in the magnetosheath but deviate some when Cluster approaches and crosses through the magnetopause. The positions of Cluster relative to each other are presented on the right-hand side of Fig. 4.

The electron flux profiles show evidence for both temporal and spatial structures. $\mathrm{C} 1$ and $\mathrm{C} 4$ are on similar paths with $\mathrm{C} 1$ being delayed by $0.78 R_{\mathrm{E}}$ or $43 \mathrm{~min}$ assuming a steady boundary. If the structure was purely spatial and steady with time, both spacecraft would observe very similar electron profiles with the measurements by $\mathrm{C} 1$ delayed in time. Although the profiles are similar, implying some spatial structure, there are significant differences meaning these are not purely spatial enhancements. The delay between when flux enhancements are observed between $\mathrm{C} 1$ and $\mathrm{C} 4$ is also far less than 43 min implying either temporal nature, a quickly outward moving boundary, or some combination of the two.

Just after 01:25 UT both $\mathrm{C} 1$ and $\mathrm{C} 4$, separated by $0.78 R_{\mathrm{E}}$, observe an enhancement at the same time implying a temporal burst. $\mathrm{C} 2$ however was just $1.11 R_{\mathrm{E}}$ away from $\mathrm{C} 4$ and 

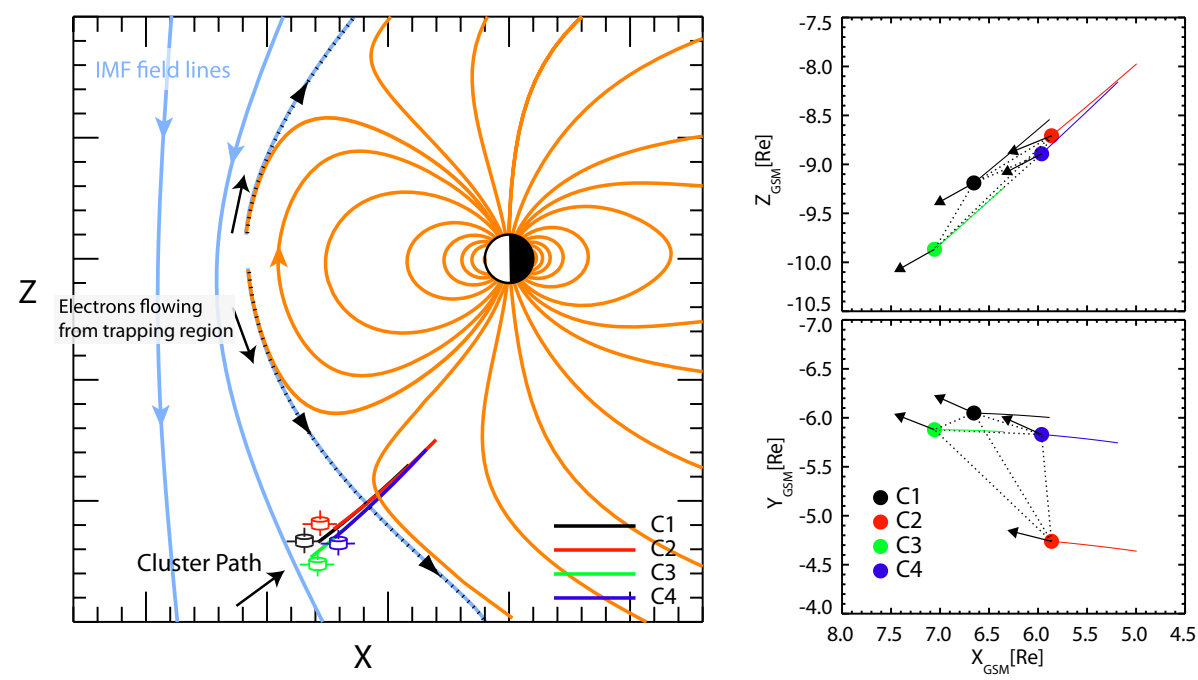

Fig. 4. A schematic diagram of the spacecraft positions and magnetic field lines for the Cluster magnetopause crossing on 3 April 2011 based on magnetic field observations is presented on the left. Dayside reconnection of the IMF (blue) and magnetospheric field lines (orange) allows trapped magnetospheric electrons to flow out onto newly opened magnetic field lines as shown by the black arrows. The right panels are the spacecraft locations and paths in GSM coordinates. The black arrows are the boundary normals identified through MVAB at 01:25 UT, and the colored traces indicate the direction the spacecraft is moving.

either did not observe the enhancement or observed it at a time just prior to $\mathrm{C} 1$ and $\mathrm{C} 4$. This means either (1) it is a temporal burst of flux but occurs in a small spatial region that may not be observed by all spacecraft, or (2) the flux enhancements are spatial and possibly confined to a boundary layer or region with motion in the GSM Y-direction that passes by $\mathrm{C} 2$ first and then by $\mathrm{C} 1$ and $\mathrm{C} 4$. If the boundary was moving past the spacecraft as outlined in option (2), the boundary normal should be primarily in the GSM Ydirection. This is not the case. The boundary normals, as identified at 01:25 UT through a minimum variance analysis of the magnetic field (MVAB) (Sonnerup and Cahill, 1967), are all consistent within $8 \%$ indicating a planar boundary at least on the size scale of the spacecraft separation. The boundary normal for each spacecraft is shown on the right side of Fig. 4 and points primarily in the X-direction. This is inconsistent with a boundary containing high energy electrons and moving primarily in the GSM Y-direction over the spacecraft. This means this burst is likely to be temporal. Although the electron profiles are similar, implying some spatial nature, there is also strong evidence for temporal effects.

\section{Discussion}

The energetic electron population along the magnetopause has been analysed here to gain understanding of the properties as well as the transport mechanisms that could populate this high-latitude region. The observations here are now compared with the expected properties for each proposed source.

\subsection{Dayside reconnection}

The process of magnetic reconnection along the magnetopause has been shown to release trapped magnetospheric electrons into the magnetosheath and along the boundary layer at lower latitudes (Dunlop et al., 2008). A possible geometry for the high-latitude magnetopause crossing on 3 April 2011 and studied here is shown on the left side of Fig. 4. This diagram shows the location of the spacecraft and the geometry of the magnetic field lines that would allow electrons released on the dayside to be observed along the high-latitude magnetopause.

One tool to test the likeliness of this mechanism is to determine if dayside reconnection is active during the time periods when the electron bursts are observed. Several probes exist to predict the occurrence of dayside reconnection. The simplest parameter to predict dayside reconnection between the solar wind and the magnetospheric is the $B_{\mathrm{Z}}$ component of the IMF (Dungey et al., 1961). During times of southward IMF, reconnection is predicted to occur more often. A number of more advanced indices have been put forth to predict the reconnection rate or the coupling between the solar wind and the magnetosphere (e.g. Perreault and Akasofu, 1978; Kan and Lee, 1979; Scurry and Russell, 1991; Newell et al., 2007). The majority of these values have been derived by assuming a functional form with solar wind parameters and computing appropriate coefficients to match geomagnetic indices such as Dst and AE. Milan et al. (2012) has assumed a functional form for the dayside reconnection rate $\Phi_{D}$ and derived coefficients through monitoring data from the IMAGE spacecraft of open and closed field line boundaries. 


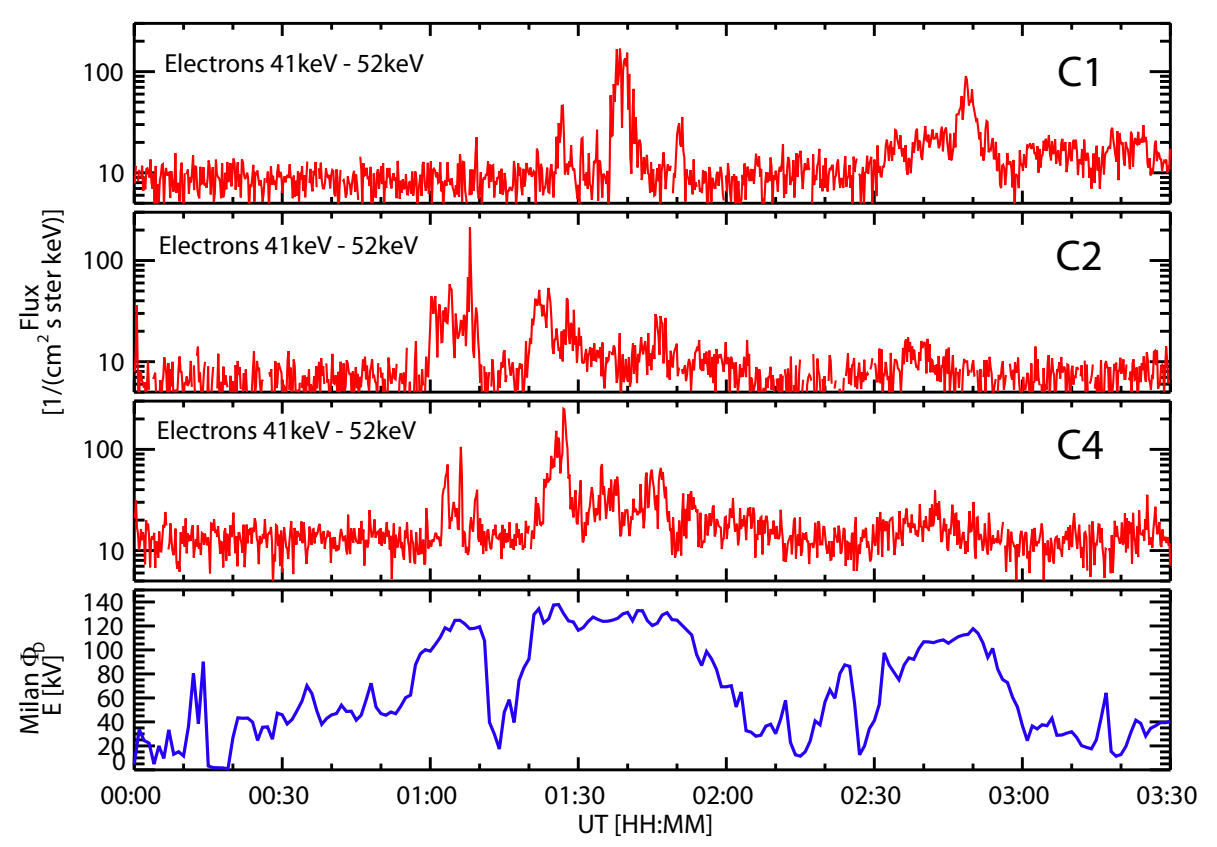

Fig. 5. The top three panels show $41-51 \mathrm{keV}$ omnidirectional electrons flux measured on $\mathrm{C} 1, \mathrm{C} 2$, and $\mathrm{C} 4$ while passing through the highlatitude magnetopause. The bottom panel is the Milan et al. (2012) reconnection potential $\Phi_{\mathrm{D}} . \Phi_{\mathrm{D}}$ is derived from upstream OMNI solar wind measurements.

Since geomagnetic indices take time to reflect energy input through dayside reconnection, measuring of open and closed field lines is a better approach to find the conditions favorable for dayside reconnection. The coupling function from Milan et al. (2012) is given in Eq. (1):

$\Phi_{\mathrm{D}}=\Lambda V_{\mathrm{x}}^{4 / 3} B_{\mathrm{YZ}} \sin ^{9 / 2}\left(\frac{1}{2} \theta\right)$

where $\Lambda$ is a constant with the value $3.3 \times 10^{5} \mathrm{~m}^{2 / 3} \mathrm{~s}^{1 / 3}, V_{\mathrm{x}}$ is the $\mathrm{X}$-component of the solar wind velocity, $B_{\mathrm{YZ}}$ is the magnitude of the $\mathrm{Y}$ and $\mathrm{Z}$ components of the IMF vector, and $\theta$ is the IMF clock angle. This $\Phi_{\mathrm{D}}$ value is compared to the electron flux to see if the bursts occur at times when one would expect dayside reconnection. The OMNI data (described at http://omniweb.gsfc.nasa.gov/) are used for upstream magnetic field and plasma parameters. Figure 5 shows the comparison of $41-52 \mathrm{keV}$ electrons measured by $\mathrm{C} 1, \mathrm{C} 2$, and $\mathrm{C} 4$ with $\Phi_{\mathrm{D}}$. The timing of each enhancement varies; however, in each case the electron flux enhancements occur during time periods of higher reconnection potential. This is consistent with the picture that electrons are streaming out on field lines newly opened through reconnection. A $40 \mathrm{keV}$ electron travels at roughly $18 R_{\mathrm{E}} \mathrm{s}^{-1}$, so little time (fractions of a second) is needed to wait for the propagation between the reconnection site and where they are observed by the Cluster spacecraft. Previous studies have shown the energetic electron fluxes to correlate with geomagnetic indices on a large time scale (hours) (Meng and Anderson, 1975; Baker and Stone, 1977), but this is the first evidence of a higher resolution correlation (one minute resolution) with a solar wind/magnetosphere coupling function.

The electron pitch angle distribution provides additional information to determine where the particles may be originating. In a broad sense, simply the direction of the energetic electron flow gives information as to the origin. If a southward pointing IMF was draped over the magnetopause and particles were released onto this field line through the reconnection site, they would travel parallel to the magnetic field line in the Southern Hemisphere. This is consistent with the observations.

In a finer sense, the location of the peak of the pitch angle distribution can give additional information. If a particle distribution peaked at a pitch angle of $90^{\circ}$ (as is often observed in the equatorial dayside magnetosphere) and travels from an area of high magnetic field strength to low magnetic field strength, the peak of the distribution will shift to become more field-aligned to conserve the first adiabatic invariant. The observations by $\mathrm{C} 2$ show a peak in pitch angle between $20^{\circ}$ and $60^{\circ}$ rather than simply a distribution peak at $0^{\circ}$. These measurements are consistent with a source such as the dayside trapping region where a particle distribution that originally peaked at $90^{\circ}$ is released to a region of lower magnetic field such as the high-latitude boundary layer.

The energy spectra and sometimes apparent bursty nature are also consistent with the geometry illustrated in Fig. 4. The figure shows the spacecraft on open magnetic field lines while crossing the high-latitude magnetopause. As the electron population is released through intermittent reconnection, 
they travel past the spacecraft in bursts. Since the spacecraft are on open field lines, the bulk of the electrons do not mirror and return. The one-directional flow is shown well through the two bursts in Fig. 2. The distribution during a burst or outside of one is relatively unchanged when looking at particles flowing antiparallel to the magnetic field line (pitch angle of $160^{\circ}-180^{\circ}$ ). When looking at electrons flowing from the direction of the dayside reconnection (pitch angle of $0^{\circ}-$ $30^{\circ}$ ), an additional population represented by a power law is present.

A recent study far downtail $\left(-72 R_{\mathrm{E}}>X_{\mathrm{GSM}}>\right.$ $\left.-102 R_{\mathrm{E}}\right)$ by the Cassini spacecraft during an Earth swing-by measured similar bursts of energetic electrons and concluded these were the result of magnetic reconnection along the flank of the magnetopause releasing trapped particles (Ogasawara et al., 2011). These bursts were short in duration and occurred while the IMF $B_{\mathrm{Z}}$ component was southward. The similarities between the electron properties measured just poleward of the cusp in the current study and far downtail by Cassini or by IMP-8 (Baker and Stone, 1978) imply the reconnection mechanism could be releasing electrons to a broad region along the magnetopause.

\subsection{Reconnection acceleration}

The process of magnetic reconnection is widely believed to be an efficient mechanism in rapidly converting magnetic energy into plasma energy. Observations from a number of spacecraft including WIND (Øieroset et al., 2002), GEOTAIL (Nagai et al., 2001) and Cluster (Vaivads et al., 2011; Åsnes et al., 2008) have presented measurements of electron acceleration up to hundreds of $\mathrm{keV}$ associated with reconnection within the magnetotail. The efficiency of reconnection to accelerate electrons in other regions however, has been less clear. Measurements of thin current sheets in a turbulent magnetosheath have shown some evidence for electron acceleration (Retinò et al., 2007), while an absence of energetic particles has been noted in reconnection in the solar wind (Gosling et al., 2005). Direct observations of particles being accelerated at the magnetopause have been limited, but simulations of the dayside magnetosphere predict it to be occurring. Modeling of this region using a particle-in-cell (PIC) model (Ding et al., 1992) and large-scale kinetic (LSK) simulations with a global MHD model (Berchem and Richard, 2008) have shown particle acceleration due to reconnection along the dayside magnetopause.

The question to now address is whether or not the observed properties by Cluster along the high-latitude magnetopause are consistent with the expected signatures of acceleration associated with reconnection. Although some plasma heating occurs in the exhaust region of an $\mathrm{X}$ line, this will only heat a plasma population to the Alfvén speed: $v_{\mathrm{A}}=B_{0} /\left(\rho \mu_{0}\right)^{1 / 2}$ where $B_{0}$ is the magnetic field strength, $\mu_{0}$ is the magnetic permeability constant, and $\rho$ is the mass density of the plasma. In the typical plasma conditions along the dayside magnetopause, this will only provide $1-2 \mathrm{keV}$ in energy to an electron, far below the energy in the observed enhancements. Additional mechanisms as part of reconnection have been put forth that are capable of heating the electrons up to the observed several hundred $\mathrm{keV}$. Two possible methods are (1) Fermi acceleration of electrons trapped within collapsing magnetic islands (Drake et al., 2006) and (2) heating of electrons passing through a reconnection electric potential across the reconnection region (Egedal et al., 2008). In both of these reconnection processes, the electrons are accelerated in a confined space. Observations in the magnetosphere have not shown the same heating for ions. This is likely due to the larger gyroradii of an ion compared to an electron. The larger gyroradii cause the ion to be scattered out of magnetic geometries an electron can travel through to gain energy. Simulations show the magnetic islands resulting from reconnection are $\sim 2500 \mathrm{~km}$ (Chen et al., 2008), while the gyroradius of a $50 \mathrm{keV} \mathrm{H}^{+}$ion is $1615 \mathrm{~km}$ in a typical magnetic field strength observed within an island. The full diameter of a gyration would be $3230 \mathrm{~km}$, which is larger than the size of the magnetic island. The result is the ion would not be able to stably gyrate and mirror inside the magnetic geometry, and therefore would scatter out without being heated as the island collapsed.

In the 3 April 2011 Cluster pass, enhanced energetic ions and electrons are observed with similar profiles implying a similar source. Since ions with energies up to hundreds of $\mathrm{keV}$ are observed, it is unlikely that acceleration within the reconnection region is causing this population. The onsets of the energetic electron and ion enhancements occur at the same time, but the ions are observed to be enhanced over a longer time period than the electrons. This is likely due to scattering of the ions along the magnetic field line due to their larger gyroradii.

\subsection{Exterior cusp}

Statistical work of electrons on the dayside of the magnetosphere shows the exterior cusp contains enhanced fluxes over a wide range of latitude (Walsh and Fritz, 2011). This population is consistently present on open field lines. The magnetic field lines threading the cusp form the magnetopause, so if these electrons leave the cusp, they could be later observed along the high-latitude magnetopause.

Modeling by Nykyri et al. (2012) has shown the presence of energetic electrons in the cusp is due to local heating via drift acceleration. As the particle gradients drift around the magnetic minimum in the cusp, they can gain energy as they travel through the electric potential created due to dayside reconnection. This heating would occur on time scales of the electron's drift period around the exterior cusp. Particle tracing by Sheldon et al. (2008) has shown this drift period to be tens of minutes. The bursts on the high-latitude magnetopause last between 30 and $120 \mathrm{~s}$. This is much shorter than the time periods of cusp drift acceleration, although if the 
trapped particles in the cusp were being released over time periods, they could be measured for the observed time periods.

The observed pitch angle distribution however is inconsistent with the energetic electrons originating in the exterior cusp. Observations (Walsh et al., 2010) and modeling (Nykyri et al., 2012) have shown electrons heated through drift acceleration in the cusp diamagnetic cavity are peaked at a pitch angle of $90^{\circ}$ in a local magnetic field minimum. Field strengths in the cusp diamagnetic cavity range between $10 \mathrm{nT}$ and $40 \mathrm{nT}$ (e.g. Farrell and Van Allen, 1990; Chen et al., 1998; Zhang et al., 2005). Particles within this magnetic field strength would not be focused on a field-aligned distribution at the observed field strengths of $43 \mathrm{nT}$ along the high-latitude magnetopause. Therefore, electrons accelerated through drift acceleration in a cusp diamagnetic cavity are not consistent with what is observed along the high-latitude magnetopause.

\section{Conclusions}

Cluster spacecraft observations have shown enhancements of energetic electrons occurring in bursts along the highlatitude magnetopause. These bursts are field-aligned flowing tailward and occur on short time scales. The bursts occur while a reconnection potential index indicates that dayside magnetic reconnection is likely to be occurring. These properties are consistent with the energetic particles being released through dayside reconnection. After being released they stream along newly opened magnetic field lines to populate the high-latitude magnetopause. The properties of the particles are more consistent with a release from the trapping region by dayside reconnection than escape from the exterior cusp or heating through reconnection acceleration. The observed particle properties are similar to those observed along the further downtail along the magnetopause, indicating that during appropriate conditions, reconnection on the dayside, or potentially along the flanks, may release particles that populate this region as well.

Acknowledgements. We would like to acknowledge the RAPID, PEACE, and FGM Cluster instrument teams for rapid handling and calibration of the data. GEOTAIL magnetic field data were kindly provided by T. Nagai. We would also like to thank M. Muskin for useful scientific discussions. Support was given by the National Science Foundation through grant AGS-1136827. The work at Boston University was supported by NASA grant NNX08AU70G.

Topical Editor I. A. Daglis thanks two anonymous referees for their help in evaluating this paper.

\section{References}

Åsnes, A., Taylor, M. G. G. T., Borg, A. L., Lavraud, B., Friedel, R. W. H., Escoubet, C. P., Laakso, H., Daly, P., and Fazakerley, A. N.: Multispacecraft observation of electron beam in reconnection region, J. Geophys. Res., 113, A12, doi:10.1029/2007JA012770, 2008.

Baker, D. N. and Stone, E. C.: The relationship of energy flow at the magnetopause to geomagnetic activity, Geophys. Res. Lett., 4, 395-398, doi:10.1029/GL004i010p00395, 1977.

Baker, D. N. and Stone, E. C.: The magnetopause energetic electron layer. I - Observations along the distant magnetotail, J. Geophys. Res., 83, 4327-4338, 1978.

Balogh, A., Dunlop, M. W., Cowley, S. W. H., Southwood, D. J., Thomlinson, J. G., Glassmeier, K. H., Musmann, G., Luhr, H., Buchert, S., Acuña, M. H., Fairfield, D. H., Slavin, J. A., Riedler, W., Schwingenschuh, K., and Kivelson, M. G.: The Cluster Magnetic Field Investigation, Space Sci. Rev., 79, 65-91, 1997.

Balogh, A., Carr, C. M., Acuña, M. H., Dunlop, M. W., Beek, T. J., Brown, P., Fornacon, K.-H., Georgescu, E., Glassmeier, K.H., Harris, J., Musmann, G., Oddy, T., and Schwingenschuh, K.: The Cluster Magnetic Field Investigation: overview of in-flight performance and initial results, Ann. Geophys., 19, 1207-1217, doi:10.5194/angeo-19-1207-2001, 2001.

Berchem, J. and Richard, R.: Magnetic reconnection and particle acceleration at the Earth's dayside magnetopause: results from global simulations, AIP Conf. Proc., 1039, 301-306, doi:10.1063/1.2982462, 2008.

Chen, J., Fritz, T. A., Sheldon, R. B., Spence, H. E., Spjeldvik, W. N., Fennell, J. F., Livi, S., Russell, C. T., Pickett, J. S., and Gurnett, D. A.: Cusp energetic particle events: Implications for a major acceleration region of the magnetosphere, J. Geophys. Res. 103, 69-78, 1998.

Chen, L.-J.; Bhattacharjee, A., Puhl-Quinn, P. A., Yang, H., Bessho, N., Imada, S., Muhlbachler, S., Daly, P. W., Lefebvre, B., Khotyaintsev, Y., Vaivads, A., Fazakerley, A., and Georgescu, E.: Observation of energetic electrons within magnetic islands, Nature, 4, 19-23, doi:10.1038/nphys777, 2008.

Daly, P. W., Saunders, M. A., Rijnbeek, R. P., Sckopke, N., and Russell, C. T.: The distribution of reconnection geometry in flux transfer events using energetic ion, plasma and magnetic data, J. Geophys. Res., 89, 3843-3854, doi:10.1029/JA089iA06p03843, 1984.

Ding, D.-Q., Lee, L.-E., and Swift, D.-W.: Particle Simulations of Driven Collisionless Magnetic Reconnection at the Dayside Magnetopause, J. Geophys. Res., 97, 8453-8481, 1992.

Domingo, V., Page, D. E., and Wenzel, K.-P.: Energetic and Relativistic Electrons Near the Polar Magnetopause, J. Geophys Res., 82, 2327-2326, 1977.

Drake, J. F., Swisdak, M., Che, H., and Shay, M. A.: Electron acceleration from contracting magnetic islands during reconnection, Nature, 443, 553-556, doi:10.1038/nature05116, 2006.

Dungey, J. W.: Interplanetary Magnetic Field and the Auroral Zones, Phys. Rev. Lett., 6, 47-48, doi:10.1103/PhysRevLett.6.47, 1961.

Dunlop, M. W., Taylor, M. G. G. T., Bogdanova, Y. V., Shen, C., Pitout, F., Pu, Z., Davies, J. A., Zhang, Q.-H., Wang, J., Lavraud, B., Fazakerley, A. N., Walsh, A., Owen, C. J., Laakso, H., Zong, Q.-G., Liu, Z.-X., Escoubet, C. P., Carr, C. M., and Rème, H.: Electron structure of the magnetopause boundary layer: Cluster/Double Star observations, J. Geophys. Res., 113, A07S19, doi:10.1029/2007JA012788, 2008. 
Egedal, J., Fox, W., Katz, N., Porkolab, M. Øieroset, M., Lin, R. P., Daughton, W., and Drake, J. F.: vidence and theory for trapped electrons in guide field magnetotail reconnection, J. Geophys. Res., 113, A12207, doi:10.1029/2008JA013520, 2008.

Farrell, W. M. and Van Allen, J. A.: Observations of the Earth's Polar Cleft at Large Radial Distances With the Hawkeye 1 Magnetometer, J. Geophys. Res., 95, 20945-20958, doi:10.1029/JA095iA12p20945, 1990.

Formisano, V. and Domingo, V.: Energetic electrons in the cusp and in the high latitude plasma sheet - Evidence for source regions, Planet Space Sci., 27, 1479-1490, doi:10.1016/00320633(79)90094-1, 1979.

Fritz, T. A., Chen, J., and Sheldon, R. B.: The Role of the Cusp as A Source for Magnetospheric Particles: A New Paradigm?, Adv. Space Res., 25, 1445-1457, doi:10.1016/S0273-1177(99)006560, 2000.

Fuselier, S. A., Anderson, B. J., and Onsager, T. G.: Particle signatures of magnetic topology at the magnetopause: AMPTE/CCE observations, J. Geophys. Res., 100, 1180511821, doi:10.1029/94JA02811, 1995.

Gosling, J. T., Skoug, R. M., McComas, D. J., and Smith, C. W.: Direct evidence for magnetic reconnection in the solar wind near $1 \mathrm{AU}$, J. Geophys. Res., 110, A01107, doi:10.1029/2004JA010809, 2005.

Johnstone, A. D., Alsop, C., Burge, S., Carter, P. J., Coates, A. J., Coker, A. J., Fazakerley, A. N., Grande, M., Gowen, R. A., Gurgiolo, C., Hancock, B. K., Narheim, B., Preece, A., Sheather, P. H., Winningham, J. D., and Woodliffe, R. D.: PEACE: A plasma electron and current experiment, Space Sci. Rev., 79, 351-398, doi:10.1023/A:1004938001388, 1997.

Kan, J. R. and Lee, L. C.: Energy coupling function and solar wind-magnetosphere dynamo, Geophys. Res. Lett., 6, 577-580, doi:10.1029/GL006i007p00577, 1979.

Kokubun, S., Yamamoto, T., Acuna, M. H., Hayashi, K., Shiokawa, K., and Kawano, H.: The Geotail magnetic field experiment, J. Geomagn. Geoelectr., 46, 7-21, 1994.

Meng, C. I. and Anderson, K. A.: A Layer of Energetic Electrons ( $>40 \mathrm{keV}$ ) near the Magnetopause, J. Geophys. Res., 75, $1827-$ 1836,1970

Meng, C.-I. and Anderson, K. A.: Characteristics of the Magnetopause Energetic Electron Layer, J. Geophys. Res., 80, 42374243, doi:10.1029/JA080i031p04237, 1975.

Milan, S. E., Gosling, J. S., and Hubert, B.: Relationship between interplanetary parameters and the magnetopause reconnection rate quantified from observations of the expanding polar cap, J. Geophys. Res., 117, A03226, doi:10.1029/2011JA017082, 2012.

Mitchell, D. G., Kutchko, F., Williams, D. J., Eastman, T. E., and Frank, L. A.: An Extended Study of the Low-Latitude Boundary Layer on the Dawn and Dusk Flanks of the Magnetosphere, J. Geophys. Res., 92, 7394-7404, doi:10.1029/JA092iA07p07394, 1987.

Nagai, T., Shinohara, I., Fujimoto, M., Hoshino, M., Saito, Y., Machida, S., and Mukai, T.: Geotail observations of the Hall current system: Evidence of magnetic reconnection in the magnetotail, J. Geophys. Res., 106, 25929-25950, doi:10.1029/2001JA900038, 2001.

Newell, P. T., Sotirelis, T., Liou, K., Meng, C.-I., and Rich, F. J.: A nearly universal solar wind-magnetosphere coupling function inferred from 10 magnetospheric state variables, J. Geophys. Res.,
112, A01206, doi:10.1029/2006JA012015, 2007.

Nykyri, K., Otto, A., Adamson, E., Kronberg, E., and Daly, P.: On the origin of high-energy particles in the cusp diamagnetic cavity, J. Atmos. Terr. Phys., in press, doi:10.1016/j.jastp.2011.08.012, 2012.

Ogasawara, K., Livi, S. A., Mitchell, D. G., Armstrong, T. P., and Krupp, N.: Properties of energetic particle bursts at dawnside magnetosheath: Cassini observations during the 1999 Earth swing-by, J. Geophys. Res., 116, A12207, doi:10.1029/2011JA016813, 2011.

Øieroset, M., Lin, R. P., Phan, T. D., Larson, D. E., and Bale, S. D.: Evidence for Electron Acceleration up to $\sim 300 \mathrm{keV}$ in the Magnetic Reconnection Diffusion Region of Earth's Magnetotail, Phys. Rev. Lett., 89, 195001, doi:10.1103/PhysRevLett.89.195001, 2002.

Perreault, P. and Akasofu, S.-I.: A study of geomagnetic storms, Geophys. J., 54, 547-573, 1978.

Peterson, W. K., Shelley, E. G., Haerendel, G., and Paschmann, G.: Energetic Ion Composition in the Subsolar Magnetopause and Boundary Layer, J. Geophys. Res., 87, 2139-2145, doi:10.1029/JA087iA04p02139, 1982.

Retinò, A., Sundkvist, D., Vaivads, A., Mozer, F., André, M., and Owen, C. J.: In situ evidence of magnetic reconnection in turbulent plasma, Nature Physics, 3, 236-238, doi:10.1038/nphys574, 2007.

Roederer, J. G.: On the adiabatic motion of energetic particles in a model magnetosphere, J. Geophys. Res., 72, 981-992, doi:10.1029/JZ072i003p00981, 1967.

Sarafopoulos, D. V., Athanasiu, M. A., Sarris, E. T., Yamamoto, T., and Kokubun, S.: Properties and origin of energetic particles at the duskside of the Earth's magnetosheath throughout a great storm, Ann. Geophys., 17, 1121-1133, doi:10.1007/s00585-9991121-2, 1999.

Sarafopoulos, D. V., Athanasiu, M. A., Sibeck, D. G., McEntire, R. W., Sarris, E. T., and Kokubun, S.: Energetic proton and electron dispersion signatures in the nightside magnetosheath supporting their leakage out of the magnetopause, J. Geophys. Res., 105, 15729-15739, doi:10.1029/2000JA900041, 2000.

Scurry, L. and Russell, C. T.: Proxy studies of energy transfer to the magnetopause, J. Geophys. Res., 96, 9541-9548, 1991.

Sheldon, R. B., Chen, J., and Fritz, T. A.: The quadrupole as a source of energetic particles: III. Outer radiation belt and MeV electrons, J. Atmos. Terr. Phys., 70, 1829-1846, doi:10.1016/j.jastp.2008.09.012, 2008.

Shprits, Y. Y., Thorne, R. M., Friedel, R., Reeves, G. D., Fennell, J., Baker, D. N., and Kanekal, S. G.: Outward radial diffusion driven by losses at magnetopause, J. Geophys. Res., 111, A11214, doi:10.1029/2006JA011657, 2006.

Sibeck, D. and McEntire, R. W.: Multiple satellite observations of leakage of particles from the magnetosphere, Adv. Space. Res., 8, 201-216, doi:10.1016/0273-1177(88)90133-0, 1988.

Sonnerup, B. U. O. and Cahill, L. J.: Magnetopause Structure and Attitude from Explorer 12 Observations, J. Geophys. Res., 72, 171-183, 1967.

Sonnerup, B. U. Ö., Paschmann, G., Papamastorakis, I., Sckopke, N., Haerendel, G., Bame, S. J., Asbridge, J. R., Gosling, J. T., and Russell, C. T.: Evidence for Magnetic Field Reconnection at the Earth's Magnetopause, J. Geophys. Res., 86, 10049-10067, doi:10.1029/JA086iA12p10049, 1981. 
Treumann, R. A., LaBelle, J., and Pottelette, R.: Plasma Diffusion at the Magnetopause: The Case of Lower Hybrid Drift Waves, J. Geophys. Res., 96, 16009-16013, doi:10.1029/91JA01671, 1991.

Vaivads, A., Retinò, A., Khotyaintsev, Yu. V., and André, M.: Suprathermal electron acceleration during reconnection onset in the magnetotail, Ann. Geophys., 29, 1917-1925, doi:10.5194/angeo-29-1917-2011, 2011.

Walsh, B. M. and Fritz, T. A.: Cluster energetic electron survey of the high-altitude cusp and adjacent regions, J. Geophys. Res., 116, A12212, doi:10.1029/2011JA016828, 2011.

Walsh, B. M., Fritz, T. A., Klida, M. M., and Chen, J.: Energetic electrons in the exterior cusp: identifying the source, Ann. Geophys., 28, 983-992, doi:10.5194/angeo-28-983-2010, 2010.

Wilken, B., Axford, W. I., Daglis, I., Daly, P., Guttler, W., Ip, W. H., Korth, A., Kremser, G., Livi, S., Vasyliunas, V. M., Woch, J., Baker, D., Belian, R. D., Blake, J. B., Fennell, J. F., Lyons, L. R., Borg, H., Fritz, T. A., Gliem, F., Rathje, R., Grande, M., Hall, D., Kecsuemety, K., McKenna-Lawlor, S., Mursula, K., Tanskanen, P., Pu, Z., Sandahl, I., Sarris, E. T., Scholer, M., Schulz, M., Söraas, F., and Ullaland, S.: RAPID: The Imaging Energetic Particle Spectrometer on Cluster, Space Sci. Rev., 79, 399-473, doi:10.1023/A:1004994202296, 1997.
Wilken, B., Daly, P. W., Mall, U., Aarsnes, K., Baker, D. N., Belian, R. D., Blake, J. B., Borg, H., Büchner, J., Carter, M., Fennell, J. F., Friedel, R., Fritz, T. A., Gliem, F., Grande, M., Kecskemety, K., Kettmann, G., Korth, A., Livi, S., McKenna-Lawlor, S., Mursula, K., Nikutowski, B., Perry, C. H., Pu, Z. Y., Roeder, J., Reeves, G. D., Sarris, E. T., Sandahl, I., Søraas, F., Woch, J., and Zong, Q.-G.: First results from the RAPID imaging energetic particle spectrometer on board Cluster, Ann. Geophys., 19, 1355-1366, doi:10.5194/angeo-19-1355-2001, 2001.

Williams, D., Fritz, T. A., Wilken, B., and Keppler, E.: An Energetic Particle Perspective of the Magnetopause, J. Geophys. Res., 84, 6385-6396, doi:10.1029/JA084iA11p06385, 1979.

Zhang, H., Fritz, T. A., and Zong, Q.-G.: Stagnant exterior cusp region as viewed by energetic electrons and ions: A statistical study using Cluster Research with Adaptive Particle Imaging Detectors (RAPID) data, J. Geophys. Res., 110, A05211, doi:10.1029/2004JA010562, 2005.

Zong, Q. G., Fritz, T. A., Korth, A., Daly, P. W., Dunlop, M., Balogh, A., Fennell, J. F., Sullivan, J. D., Friedel, R. W. H., and Reme, H.: Energetic electrons as a field line topology tracer in the high latitude boundary/cusp region: Cluster/RAPID observations, Surv. Geophys., 26, 2150-240, 2005. 